\title{
Numerical simulation of Lewis number effects on lean premixed turbulent flames
}

\author{
John B. Bell, Robert K. Cheng Marcus S. Day, Ian G. Shepherd
}

Lawrence Berkeley National Laboratory, Berkeley, CA 94720, USA

\begin{abstract}
A dominant factor in determining the burning rate of a premixed turbulent flame is the degree to which the flame front is wrinkled by turbulence. Higher turbulent intensities lead to greater wrinkling of the flame front and an increase in the turbulent burning rate. This picture of turbulent flame dynamics must be modified, however, to accommodate the affects of variations in the local propagation speed of the flame front. Classical flame analysis characterizes these local variations in propagation speed by the Markstein number which represents the response of the flame front to curvature and strain. In this paper, we consider lean premixed flames for three different fuels having widely varying fuel Lewis numbers corresponding to widely varying Markstein numbers. In particular, we present numerical simulations of premixed turbulent flames for lean hydrogen, propane and methane mixtures in two dimensions. Each simulation is performed at turbulence conditions similar to those found in laboratory-scale experiments and is performed using detailed chemical kinetics and transport properties. We discuss the effect of Lewis number on the overall flame morphology and explore the dependence of local flame propagation speed on flame curvature. We also explore the relationship between local flame speed and experimentally accessible variables such as $\mathrm{OH}$ concentration. Finally, we focus on the low Lewis number case, hydrogen, in which the flame front is broken indicating local extinction.
\end{abstract}

Keywords: turbulent premixed combustion, low Mach number flow, adaptive mesh refinement 


\section{Introduction}

Recent interest in alternative fuels such as hydrogen or syngas, obtained from coal gasification, has sparked interest in the development of burners that can operate over a broad range of fuels. There is also an increasing interest in lean-premixed systems that have the potential for the high efficiency and low pollutant emissions that result from lower burnt-gas temperatures. The feasilibity of lean premixed fuelflexible systems depends on the development of robust flame stabilization techniques. One issue that must be addressed when dealing with a variety of fuels is the possible impact of thermo-diffusive instabilities on the overall flame dynamics. To investigate this issue we have performed a numerical study of Lewis number effects on premixed turbulent flame dynamics characteristic of laboratory-scale experiments. Twodimensional simulations were conducted at lean conditions for propane, methane and hydrogen using detailed chemistry and transport, corresponding to $L e>$ $1, L e \approx 1$ and $L e<1$, respectively.

Since our goal is to look at detailed chemical and transport effects on the flame, we use a twodimensional idealized configuration instead of a realistic three-dimensional burner. The configuration we use is a modified version of one frequently employed in the numerical study of premixed turbulent flames. A flat steady laminar premixed flame is initialized in the computational domain and allowed to propagate toward the inflow boundary where turbulent perturbations have been superimposed on the mean flow. The turbulent fluctuations are chosen to match the turbulent intensity and integral scale of the corresponding experiment. Here, we use a feedback control algorithm that automatically adjusts the reactant flow rate to stabilize the flame above the inflow face [1]. The simulation is run until the flame is stabilized, after which statistics are gathered from the subsequent evolution.

There is an extensive literature on computational studies of this type of configuration without automatic control, both with simplified and detailed chemistry. Two-dimensional examples germane to this configuration include Baum et al. [2] who studied turbulent flame interactions for detailed hydrogen chemistry and more recent studies by Chen and Im [3] and Im and Chen [4]. Haworth et al. [5] examined the effect of inhomogeneous reactants for propane/air flames using detailed propane chemistry at conditions typical of an IC engine. Analogous studies in 3D have been performed by Rutland and Trouvé [6], Trouvé and Poinsot [7], Zhang and Rutland [8], and Chakraborty and Cant [9]. All of these 3D studies were based on simplified chemistry. More recently Tanahashi et al. $[10,11]$ performed simulations of this type for turbulent premixed hydrogen flames with detailed hydrogen chemistry. Bell et al. [12] performed a similar study for a turbulent methane flame. The simulations reported here, performed using a low Mach number model, cover a physical domain significantly larger than previous calculations and thereby can represent the large range of eddies found in laboratory flames.

In the next section, we summarize the basic simulation methodology for low-speed reacting flows and the feedback control procedure, and then discuss the computations in detail. In the subsequent section, we quantify the variation in local flame properties for the different fuels. We also discuss the relationship between standard flame observable variables and local flame speeds, with particular emphasis on issues related to the hydrogen case where the flame front is discontinuous.

\section{Computational Methodology}

\subsection{Low Mach number model}

The simulations presented here are based on a low Mach number formulation of the reacting flow equations. The methodology treats the fluid as a mixture of perfect gases. We use a mixture-averaged model for differential species diffusion and ignore Soret, Dufour, gravity and radiative transport processes. With these assumptions, the low Mach number equations for an open domain are

$$
\begin{aligned}
& \frac{\partial \rho U}{\partial t}+\nabla \cdot \rho U U=-\nabla \pi+\nabla \cdot \tau, \\
& \frac{\partial \rho Y_{m}}{\partial t}+\nabla \cdot U \rho Y_{m}=\nabla \cdot \rho D_{m} \nabla Y_{m}-\dot{\omega}_{m}, \\
& \frac{\partial \rho h}{\partial t}+\nabla \cdot U \rho h=\nabla \cdot \frac{\lambda}{c_{p}} \nabla h+ \\
& \sum_{m} \nabla \cdot h_{m}\left(\rho D_{m}-\frac{\lambda}{c_{p}}\right) \nabla Y_{m},
\end{aligned}
$$

where $\rho$ is the density, $U$ is the velocity, $Y_{m}$ is the mass fraction of species $m, h$ is the mass-weighted enthalpy of the gas mixture, $T$ is the temperature, and $\dot{\omega}_{m}$ is the net destruction rate for species $m$ due to chemical reactions. Also, $\lambda$ is the thermal conductivity, $\tau$ is the stress tensor, $c_{p}$ is the specific heat of the mixture, and $h_{m}(T)$ and $D_{m}$ are the enthalpy and species mixture-averaged diffusion coefficients of species $m$, respectively. These evolution equations are supplemented by an equation of state for a perfect gas mixture:

$$
p_{0}=\rho R_{m i x} T=\rho \mathcal{R} T \sum_{m} \frac{Y_{m}}{W_{m}}
$$

where $W_{m}$ is the molecular weight of species $m$, and $\mathcal{R}$ is the universal gas constant. In the low Mach number model, the equation of state constrains the evolution and removes acoustic wave propagation from the dynamics of the system.

The basic discretization combines a symmetric operator-split treatment of chemistry and transport with a density-weighted approximate projection method. The projection method incorporates the constraint by imposing a constraint on the velocity divergence that forces the evolution to satisfy the equation of state. The resulting integration of the advective 
terms proceeds on the time scale of the relatively slow advective transport. Faster diffusion and chemistry processes are treated time-implicitly. This integration scheme is embedded in a parallel adaptive mesh refinement algorithm framework based on a hierarchical system of rectangular grid patches. The complete integration algorithm is second-order accurate in space and time, and discretely conserves species mass and enthalpy. The reader is referred to [13] for details of the low Mach number model and its numerical implementation and to [12] for previous applications of this methodology to the simulation of premixed turbulent flames.

\subsection{Control algorithm}

The flow configuration we consider initializes a flat laminar flame in a domain oriented so that the flame propagates downward. Since no gravitational force is included, up and down are for orientation only. A cold fuel-air premixture enters the domain through bottom boundary, and hot combustion products exit the domain through the top. The remaining computational boundary is periodic. Along the inflow face we specify both a mean inflow velocity and turbulent fluctuations (discussed in more detail below) that are superimposed on the mean inflow. A control algorithm, introduced in Bell et al. [1], automatically adjusts the mean inflow rate to hold the flame at a fixed location in the domain. impose induce (See [1] for additional details and a demonstration of the efficacy of the control algorithm applied to premixed flames.)

\subsection{Case study}

We consider three fuels, propane, methane and hydrogen, all at lean conditions at $1 \mathrm{~atm}$ with an ambient temperature of $298 \mathrm{~K}$. For methane and hydrogen, we used GRI-Mech 1.12 for the chemical kinetics, thermodynamics and transport. Propane was treated using the San Diego mechanism (release 20050310) [14]. Equivalence ratios were taken to match conditions for which experimentla data are available. Detailed properties of the flames are presented in Table 1.

Each of the flames is simulated on a domain that is $8 \mathrm{~cm}$ wide and $16 \mathrm{~cm}$ in height. Turbulent perturbations to the velocity field enter the domain at the lower boundary. The turbulence is generated on an $8 \mathrm{~cm} \times$ $8 \mathrm{~cm}$ domain with a prescribed energy spectrum:

$$
E(k)=\frac{\left(\frac{k}{k_{i}}\right)^{4}}{\left[1+\left(\frac{k}{k_{i}}\right)^{2}\right]^{\frac{17}{6}}} e^{-\frac{9}{4}\left(\frac{k}{k_{d}}\right)^{\frac{4}{3}}}
$$

where $k$ is the wavenumber, $k_{d}=6400 \mathrm{~cm}^{-1}$ and $k_{i}=65 \mathrm{~cm}^{-1}$. The resulting random field is then evolved using an incompressible flow solver to adjust the phasing of the velocity fields. The resulting synthetic turbulence is isotropic with an integral scale $\ell_{t}=0.3 \mathrm{~mm}$ and a turbulent intensity, $u^{\prime}=18.5$ $\mathrm{cm} / \mathrm{s}$, again matching conditions used in experiment.

The simulations were performed with adaptive mesh refinement using base mesh of $160 \times 320$. Refinement was based on two criteria. First, we refined on vorticity when

$$
|\nabla \times U|>\omega_{e r r} \times 2^{\ell-1}
$$

for levels $\ell=1,2,3$ where $\omega_{\text {err }}=100$ so that increasingly intense vortices received higher resolution up to level $\ell=4$ with an effective resolution of $1280 \times 2560$ for vortices of magnitude greater than 400. In addition, we forced refinement around the flame front by refining on high concentrations of a specific flame marker. For the propane and methane flames $\mathrm{CH}_{3}$ was used as the flame marker and $\mathrm{H}$ was used to mark the hydrogen flame. For the hydrogen case, four adaptive refinement levels were found to resolve adequately the flame with an effective resolution of $\Delta x=62.5 \mu \mathrm{m}$. The flame structures in both the hydrocarbon cases were thinner than that of the hydrogen case; an additional level of refinement was introduced at the flame front, for an effective resolution of $\Delta x=31.25 \mu \mathrm{m}$. Each of the flames was evolved until the mean height of the flame above the inlet boundary became statistically stationary prior to collecting data for further analysis.

\section{Analysis of Flames}

\subsection{Appearance of the Flames}

Representative snapshots of the mole fraction of $\mathrm{OH}$ for the three flames are shown in Figure 1. These may be compared with experimental OH PLIF measurements taken using the low swirl burner introduced by Bedat and Cheng [15] shown in Figure 2. For methane and propane, the equivalence ratio in the simulation is the same as was used in the experiment; for hydrogen we needed to increase the equivalence ratio from $\phi=0.27$ used in the experiments to $\phi=0.37$ in order to compensate for the detailed hydrogen mechanism's poor prediction of the laminar flame speed at ultra lean conditions. (Several other mechanims were tested but none were found that produced satisfactory results at such lean conditions.)

At any instant in time, the flame surfaces show the characteristic wrinkling expected of a turbulent premixed flame, namely, smooth regions where the flame is convex toward the reactants separated by sharper cusps protruding into the burned region. The size of the region imaged in the experimental PLIF measurements is indicated by the rectangles on the images in Figure 1 . We note that although the simulations were performed in two dimensions, the PLIF images indicate that the experiment and the simulations exhibit qualitatively similar levels of wrinkling for each of the fuels considered.

Comparing the images for the different fuels, we see that the propane flame, where $L e>1$, shows the smallest degree of wrinkling. In this flame, peaks in the $\mathrm{OH}$ profile coincide with cusps in the flame surface, and the cusps are always spiked toward the products and bowed toward the reactants. The methane flame, with $L e \approx 1$, exhibits a somewhat larger degree of wrinkling, but also considerably less $\mathrm{OH}$ variation along the flame front. The hydrogen flame is 
thermo-diffusively unstable and is therefore considerably more distorted than the other two. The behavior of $\mathrm{OH}$ is the converse of the other two flames, peaking in the bowed regions. At the lean conditions considered here, the images of the hydrogen flame show breaks in the $\mathrm{OH}$ profile (features which are widely believed to indicate local flame extinction). We note that a flame front position defined in terms of an isotherm (Table 1) extends across breaks in the $\mathrm{OH}$ signal.

\subsection{Global Burning Speed}

For the initial analysis, we look first at a global burning speed, $S_{c}^{G}$, defined in terms of the fuel consumption rate integrated over the entire domain at each instant in time

$$
S_{c}^{G}=\frac{1}{A_{L}\left(\rho Y_{\text {Fuel }}\right)_{\text {in }}} \int_{\Omega} \rho \omega_{\text {Fuel }} d \Omega
$$

Here $A_{L}$ is the width of the domain, $L,\left(\rho Y_{F u e l}\right)_{\text {in }}$ is the inflowing fuel mass density and $\rho \omega_{F u e l}$ is the rate of fuel consumption. We also define the instantaneous flame area $A_{G}$ to be the length of a contour defining the flame.

Figure 3 shows a scatter plot of $S_{c}^{G} / S_{L}$ versus $A_{G} / A_{L}$ for the propane and hyrdogen flames. (We have not plotted data for the methane flame; it is well known that for methane at $\phi=0.7-0.8$ the instantaneous global burning speed is accurately approximated the the laminar flame speed $\times$ the wrinkled flame area. See, for example, [1].) For the plots in Fig. 3, we considered two different isotherms for the definition of $A_{G}$ : the isotherm of peak fuel consumption, and the $T=650 \mathrm{~K}$ isotherm (which is similar to what would be observed in Mie scattering images from oil droplets). For propane, the two isotherms give similar results. The data shows the expected trend but the actual flame speed is somewhat underpredicted by the area, reflecting the intense burning at negative curvature. For hydrogen, the $T=650 \mathrm{~K}$ isotherm provides a good correlation between area and global flame speed but $S_{c}^{G}$ is substantially higher than $S_{L} \times A_{G}$. For the $T=1144 \mathrm{~K}$ isotherm, the data provides a relatively poor correlation because the shape of the isotherm in the extinction pockets, as seen in Figure $3 \mathrm{c}$, is determined by thermal conduction and is unrelated to chemical reactions.

\subsection{Local Burning Speed Behavior}

The variation in Lewis number of the three flames we consider results in differing responses of the flames to wrinkling as characterized by the Markstein number. See, for example [16]. Here, we want to examine variations in local flame speed as a function of curvature. There are several potential definitions of local flame speed; see, e.g., Poinsot and Veynante [17] for a discussion of possible choices. Here we will define a local flame speed based on integrated local fuel consumption. Figure 4 depicts the local fuel consumption in a sub-region of the domain and serves to illustrate the response of the different fuels to wrinkling.

To specify the integrated local conumption flame speed, we first identify the flame with the isotherm at which fuel consumption reaches its maximum in the corresponding flat unstretched laminar flame (Table 1). We then define a local coordinate system near the flame using arc length along the flame and a normal coordinate defined in terms of temperature scaled to define a progress variable, $c$, such that $c=0$ in the unburned reactants, and $c=1$ in the products.

At uniform intervals along the flame, we follow integral curves of the gradient of $c$ toward both the products and fuel, defining a series of adjacent disjoint wedge-shaped volumes, $\Omega_{k}$, surrounding the flame, and extending well beyond the region of high chemical reactivity. A local burning speed may then be defined over each of these volumes:

$$
S_{c}^{\ell}=\frac{1}{A^{\ell}\left(\rho Y_{\text {Fuel }}\right)_{\text {in }}} \int_{\Omega_{k}} \rho \omega_{\text {Fuel }} d \Omega_{k}
$$

where $A^{\ell}$ is the area (length) of the intersection of $\Omega_{k}$ with the flame. For additional detail about these constructions see [1].

The curvature is defined as $\kappa=\nabla \cdot \hat{n}$ where $\hat{n}=-\nabla T /\|\nabla T\|$. (Note these normals have been chosen to point toward the cold, unburned mixture.) Curvature is evaluated in a region around the flame using centered differences and then interpolated to the isotherm we identify with the flame surface. With this definition, the curvature is negative at cusps and positive in the regions that are convex with respect to the reactants.

In Figure 5 we plot the local burning flame speed versus curvature for each of the flames. For the propane flame we observe a strong negative correlation reflecting the enhanced fuel consumption in regions of negative curvature. This correlation reflects a positive Markstein number indicative of a thermodiffusively stable flame. A similar but much weaker correlation is observed for the methane flame indicating a smaller but positive Markstein number. The correlation structure for hydrogen is considerably different. The data shows a very strong positive correlation with positive curvature and a weaker positive correlation with negative curvature. These correlations arise from the thermodiffusive instability of the flame. Also note that the flame is extinguished in some regions. In the next section, we look in more detail at these extinction events.

Before examining local extinction events in the hydrogen flame it is of interest to look at the correlation of peak $\mathrm{OH}$ mole fraction with local burning speed. Experimentally, it is not possible to directly measure a local flame speed based on fuel consumption. Experimentalists often use OH PLIF measurements to image the flame. In Figure 6 we show a correlation between the local burning speed and the peak mole fraction of $\mathrm{OH}$ in the integral region used to define the local burning speed discussed above. From the figure, 
we can see that $\mathrm{OH}$ provides an excellent marker for the flames. Furthermore, by using these correlations, one can potentially directly relate $\mathrm{OH}$ signal intensity to the local fuel consumption.

\section{Extinction events in hydrogen}

A dominant feature of the hydrogen flame in both experiment (see Fig. 2 and Chen and Bilger [18]) and the simulations is the existence of regions of local flame extinction. In this section, we characterize the dynamics of these extinction events. In Figure 7 we plot a time sequence of fuel consumption overlaid with streamlines of the instantaneous velocity field showing the formation of an extinction pocket. Although the local burning speed correlates strongly with curvative for the hydrogen flame, this extinction event begins in a region of positive curvature. The velocity field on the cold (lower) side of the flame in the initial frame indicates an expansive strain with respect to the flame surface. As the flame is stretched, it begins to weaken and recede downstream due to a reduced local burning speed. This pattern continues to develop in time until the flame sheet finally breaks (ie., $\mathrm{H}_{2}$ consumption drops significantly). We note that when the break occurs, the unburned fluid is channeled into a region of flow convergence with strongly burning regions of positive curvature on either side of the channel.

Next, we consider in more detail what is occuring in both the extinction pocket and in the strongly burning regions on either side. To quantify the behavior we follow parcels of fluid as they pass through the flame, and decompose the change in composition of each parcel into chemical and diffusive transport components. Here, we consider two such space-time trajectories (pathlines), one passing through an extinction gap and the other passing through a strongly burning region nearby. We then use the space-time coordinates of these pathlines to interrogate the simulation data. The locations where these two pathlines pass through the flame are shown in Figure 7e. In both cases, all of the fuel $\left(\mathrm{H}_{2}\right)$ disappears as the path enters the post flame region. The principal reaction that oxidizes $\mathrm{H}_{2}$ at this equivalence ratio is $\mathrm{OH}+\mathrm{H}_{2} \rightarrow \mathrm{H}+\mathrm{H}_{2} \mathrm{O}$ with a smaller contribution ( $5-7 \times$ smaller) from the chain branching reaction $\mathrm{O}+\mathrm{H}_{2} \rightarrow \mathrm{H}+\mathrm{OH}$. However, analysis of the composition change along these pathlines shows that for the path going through the extinction region, almost $97 \%$ of the $\mathrm{H}_{2}$ is diffusively transported out of the fluid. By contrast, the available $\mathrm{H}_{2}$ on the other pathline increases by approximately $72 \%$ from diffusive transport into that fluid parcel. This disparity is reflected in a factor of more than 50 in fuel consumption between the two pathlines. The dynamics here is a direct consequence of the high diffusion coeffient of $\mathrm{H}_{2}$ relative to thermal conduction. As reactants move into an extinction region $\mathrm{H}_{2}$ rapidly diffuses toward the two strong reaction zones on either side of the region to that the fuel is depleted before the slower thermal conduction can heat the reactants enough to ignite.

An examination of the simulation data shows that these structures in the flow are, in fact, reasonably robust. Once they have formed, these extinguished regions do not appear to reignite. Instead, they merge together in two somewhat distinct ways. The first is illustrated in Figure 8, which shows the merger of two extinction pockets. The dynamics here is similar to the merger of Landau Darrieus cusps in that the two pockets smoothly approach each other, eventually eliminating the burning region in between. The other type merger results from larger-scale distortions of the flame that envelope a segment of the flame containing many extinction pockets. These larger distortions typically evolve into long, thin protusions on unburnt hydrogen extending into the products. In some cases, the dynamics can lead to a pinch off creating a detached pocket. In either case, the fuel in the protusion is then consumed and the structure collapses into a single extinction pocket. In Figure 1 one of these larger scale events is in the process of occuring near the center of the hydrogen flame.

\section{Conclusions}

In this paper, we have examined the effects of Lewis number on lean premixed combustion using, primarily, two-dimensional simulations of hydrogen, methane and propane flames. The conditions for the simulations were chosen to match conditions near the core of a low swirl burner and comparisons with experimental data suggest the simulations capture the basic structure of the flames. As expected local burning rate in the methane flame is relatively insensitive to the flame curvature while the propane and hydrogen flames show strong sensitivities. For the propane flame the burning is enhanced in regions of large negative curvature indicating that the flame is thermodiffusively stable. For the thermo-diffusively unstable hydrogen flame burning is enhanced in regions of positive curvature and shows pockets of local extinction when curvature is negative. This behavior leads to the formation of breaks in the flame surface that are flanked by positively curved regions of strong burning. These structures are relatively stable; the extinction regions do not reignite. Instead they participate in merger events in which two or more extinction pockets merge to form a single pocket. Analysis of the behavior along a pathline passing through one of the gaps in the flame shows that the fuel diffuses away from the rgion into the strongly burning regions that flank the extinction pocket.

\section{Acknowledgments}

This research used resources of the National Center for Computational Sciences at Oak Ridge National Laboratory, which is supported by the Office of Science of the U.S. Department of Energy under Contract No. DE-AC05-00OR22725. The authors were supported by the Office of Science through the Office 
of Advanced Scientific Computing Research, Mathematical, Information, and Computational Sciences Division under U.S. Department of Energy contract DE-AC03-76SF00098.

\section{References}

[1] J. B. Bell, M. S. Day, J. F. Grcar, M. J. Lijewski, Accepted, Comm. App. Math. Comput. Sci.

[2] M. Baum, T. J. Poinsot, D. C. Haworth, N. Darabiha, J. Fluid Mech. 281 (1994) 1-32.

[3] J. H. Chen, H. Im, Proc. Combust. Inst. 28 (2000) 211218.

[4] H. G. Im, J. H. Chen, Combust. Flame 131 (2002) 246258.

[5] D. C. Haworth, R. J. Blint, B. Cuenot, T. J. Poinsot, Combust. Flame 121 (2000) 395-417.

[6] C. J. Rutland, A. Trouvé, Combust. Flame 94 (1993) $41-57$.

[7] A. Trouvé, T. J. Poinsot, J. Fluid Mech. 278 (1994) $1-31$.

[8] S. Zhang, C. J. Rutland, Combust. Flame 102 (1995) 447-461.

[9] N. Chakraborty, S. Cant, Combust. Flame 137 (2004) 129-147.

[10] M. Tanahashi, M. Fujimura, T. Miyauchi, Proc. Combust. Inst. 28 (2000) 529-535.

[11] M. Tanahashi, Y. Nada, Y. Ito, T. Miyauchi, Proc. Combust. Inst. 29 (2002) 2041-2049.

[12] J. B. Bell, M. S. Day, J. F. Grcar, Proc. Combust. Inst 29 (2002) 1987-1993.

[13] M. S. Day, J. B. Bell, Combust. Theory Modelling 4 (2000) 535-556.

[14] Chemical-Kinetic Mechanisms for Combustion Applications, http://maeweb.ucsd.edu/ combustion/, Center for Energy Research (Combustion Division), University of California at San Diego.

[15] B. Bedat, R. K. Cheng, Combust. flame 100 (1995) 485-494.

[16] N. Peters, Turbulent Combustion, Cambridge University Press, Cambridge, 2000.

[17] T. Poinsot, D. Veynante, Theoretical and Numerical Combustion, R. T. Edwards, Inc., Philadelphia, 2001.

[18] Y. C. Chen, R. W. Bilger, Combust. Flame 138 (2004) 155-174.
Table 1: Laminar characteristics of the three flames at equivalence ratio, $\phi$ : Thermal thickness, $\delta=\left(T_{\text {prod }}-\right.$ $\left.T_{\text {fuel }}\right) / \max \|\nabla T\|(\mu \mathrm{m})$, laminar burning speed, $s_{L}$ $(\mathrm{m} / \mathrm{s})$, and temperature $\left(T_{\max } \mathrm{K}\right)$ and $\mathrm{OH}$ mole fraction $\left(\mathrm{OH}_{\max }\right)$ at the location of peak heat release.

\begin{tabular}{l|c|c|c|c|c} 
Fuel & $\phi$ & $\delta$ & $s_{L}$ & $T_{\max }$ & $\mathrm{OH}_{\max }$ \\
\hline $\mathrm{C}_{3} \mathrm{H}_{8}$ & 0.70 & 561 & 20.6 & 1366 & .00473 \\
$\mathrm{CH}_{4}$ & 0.80 & 521 & 26.2 & 1571 & .00583 \\
$\mathrm{H}_{2}$ & 0.37 & 797 & 15.1 & 1144 & .00118
\end{tabular}



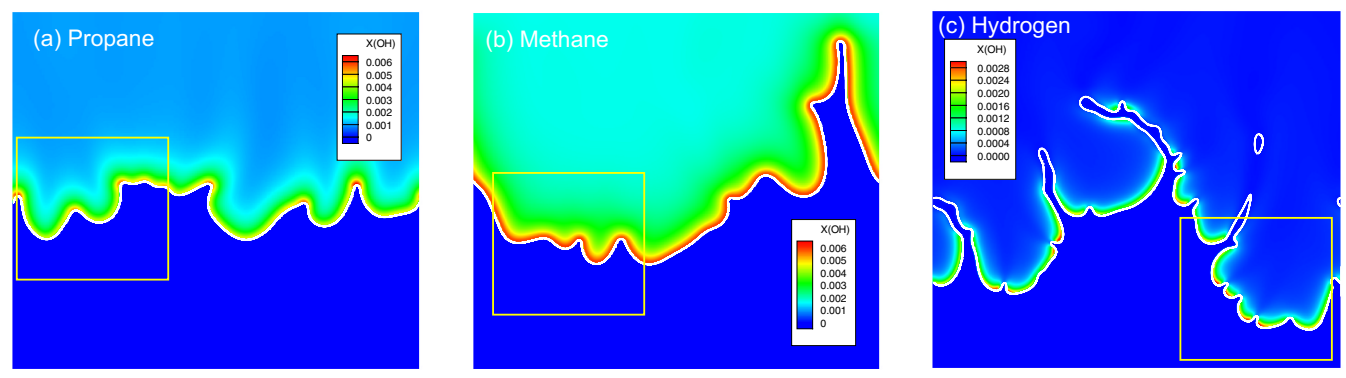

Fig. 1: Mole fraction of $\mathrm{OH}$ from representative snapshots of each flame. The temperature contour, $T=T_{\max }$ (see Table 1) is drawn over the image to indicate the flame location, and the yellow box indicates the physical extent of the corresponding PLIF images in Figure 2. The image widths correspond to $8 \mathrm{~cm}$.
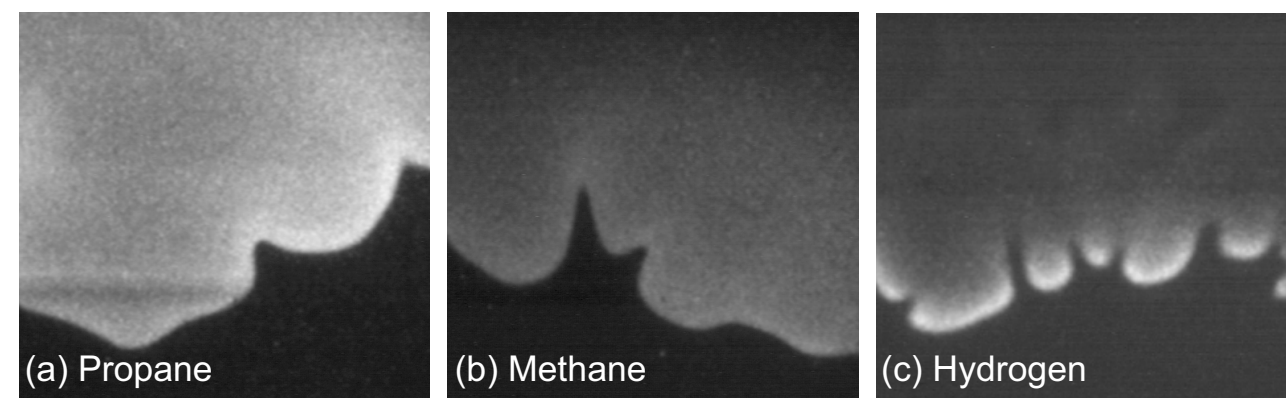

Fig. 2: Typical experiment OH PLIF for each fuel. The image widths correspond to $3 \mathrm{~cm}$.
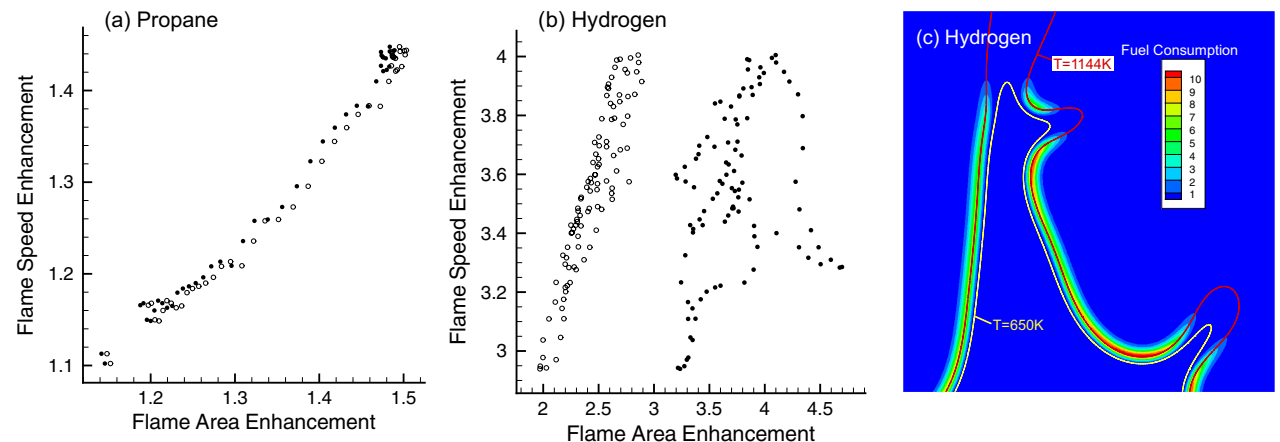

Fig. 3: Global fuel consumption (normalized by laminar burning speed) vs. flame length (normalized by domain width-i.e. length of flame laminar flame), for (a) propane, and (b) hydrogen. Data corresponding to the open symbols was computed from the $\mathrm{T}=650 \mathrm{~K}$ isocontours. The filled symbols represent data computed at the temperature of peak heat release in the corresponding flame laminar flame (see Table 1). (c) Fuel consumption $\left(\mathrm{g} /\left(\mathrm{cm}^{3} \mathrm{~s}\right)\right.$ ) near the hydrogen flame surface, overlaid with contours of $T=650 \mathrm{~K}$ and $T=T_{\max }$. 

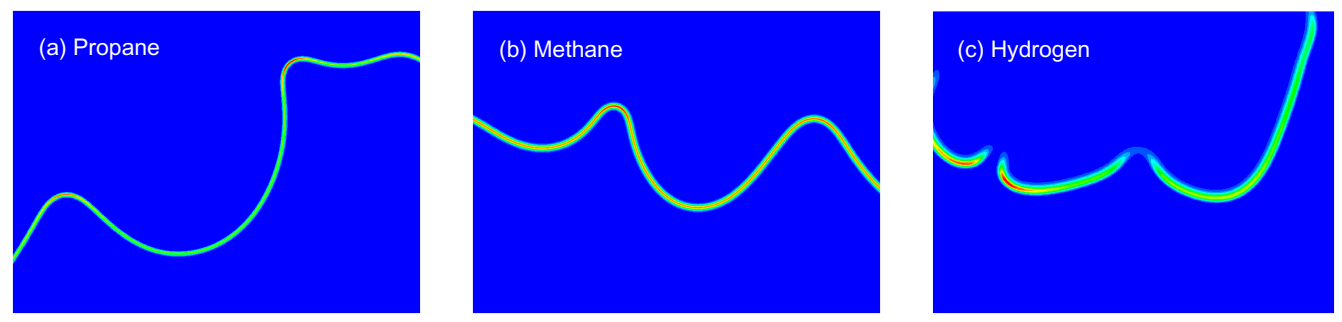

Fig. 4: Typical fuel consumption rate. The image widths correspond to $1.2 \mathrm{~cm}$.
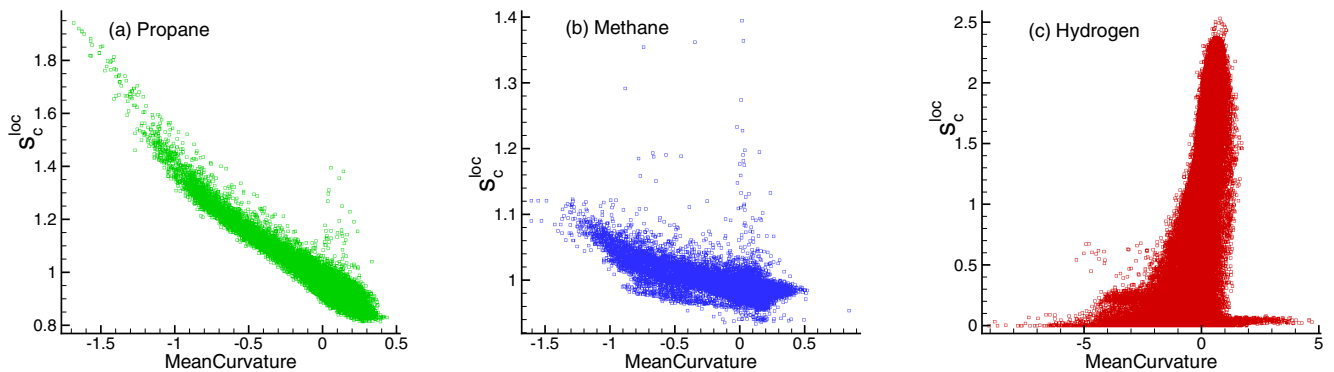

Fig. 5: Correlation of local fuel consumption flame speed with curvature for each flame. Flame speeds are normalized to corresponding laminar burning speed and curvature is normalized with the laminar flame thermal thickness (see Table 1).
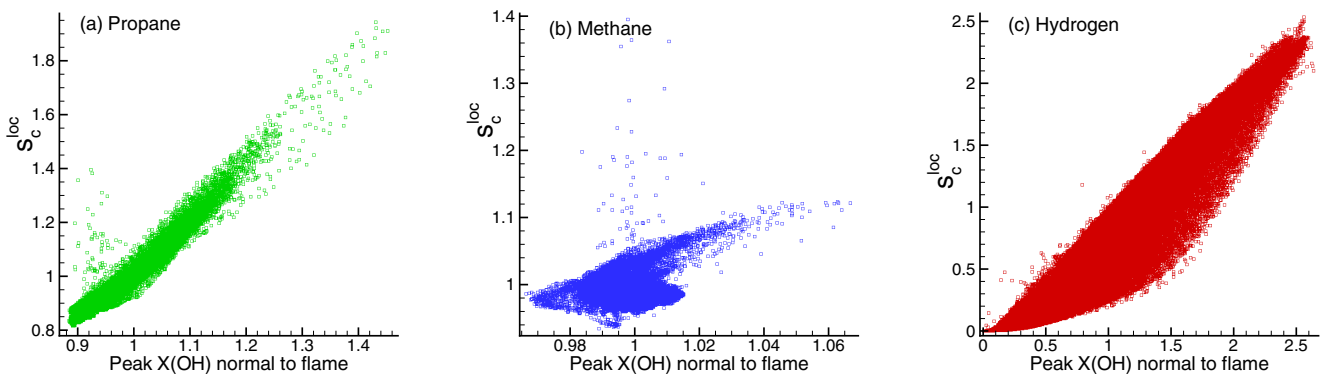

Fig. 6: Correlation of local fuel consumption flame speed with peak $\mathrm{OH}$ mole fraction for each flame. Flame speeds are normalized to corresponding laminar burning speed (see Table 1), and $\mathrm{OH}$ mole fractions are scaled by the flat laminar flame peak values.
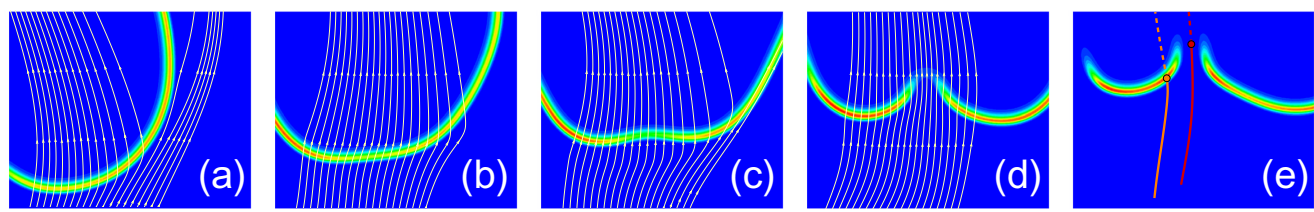

Fig. 7: Formation of an extinction pocket, as depicted by the local instantaneous fuel consumption rate. The images correspond to a subregion of the domain approximately $16 \mathrm{~mm}$ wide, and are snapshots taken at intervals of approximately 3-5 msec. The arrows overlaid on plots (a)-(d) represent streaklines, or paths through the instantaenous velocity field. Lines overlaying plot (e) represent the path taken in the Lagrangian analysis discussed in the text, where the red path is chosen to pass through the point of extinction at the time of the last frame here. The orange path is chosen to pass through the nearby peak in fuel consumption at this same instant in time. The dotted portions of both paths are traversed by the diagnostic AFTER the final frame shown here. 

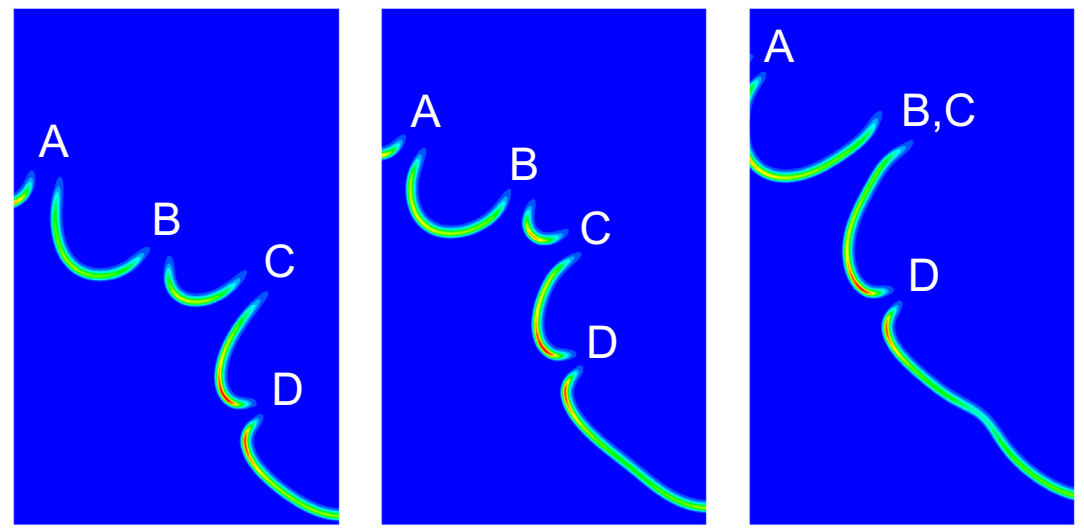

Fig. 8: Images of fuel consumption rate (same scale as Fig. 3c) in the premixed hydrogen flame showing the merger of two extinction pockets. The image widths correspond to $15.3 \mathrm{~mm}$, and represent intervals in time separated by approximately $4 \mathrm{~ms}$. 